Louisiana State University

LSU Digital Commons

Faculty Publications

Department of Geology and Geophysics

$12-15-2015$

\title{
Giant fossil mass wasting off the coast of West India: The Nataraja submarine slide
}

\author{
Gérôme Calvès \\ Universite Paul Sabatier Toulouse III \\ Mads Huuse \\ The University of Manchester \\ Peter D. Clift \\ Louisiana State University \\ Stéphane Brusset \\ Universite Paul Sabatier Toulouse III
}

Follow this and additional works at: https://digitalcommons.Isu.edu/geo_pubs

\section{Recommended Citation}

Calvès, G., Huuse, M., Clift, P., \& Brusset, S. (2015). Giant fossil mass wasting off the coast of West India: The Nataraja submarine slide. Earth and Planetary Science Letters, 432, 265-272. https://doi.org/ 10.1016/j.epsl.2015.10.022

This Article is brought to you for free and open access by the Department of Geology and Geophysics at LSU Digital Commons. It has been accepted for inclusion in Faculty Publications by an authorized administrator of LSU Digital Commons. For more information, please contact ir@lsu.edu. 
archives-ouvertes

\title{
Giant fossil mass wasting off the coast of West India: The Nataraja submarine slide
}

\author{
Gérôme Calvès, Mads Huuse, Peter Clift, Stéphane Brusset
}

\section{To cite this version:}

Gérôme Calvès, Mads Huuse, Peter Clift, Stéphane Brusset. Giant fossil mass wasting off the coast of West India: The Nataraja submarine slide. Earth and Planetary Science Letters, Elsevier, 2015, 10.1016/j.epsl.2015.10.022 . hal-01242470

HAL Id: hal-01242470

\section{https://hal.archives-ouvertes.fr/hal-01242470}

Submitted on 12 Dec 2015

HAL is a multi-disciplinary open access archive for the deposit and dissemination of scientific research documents, whether they are published or not. The documents may come from teaching and research institutions in France or abroad, or from public or private research centers.
L'archive ouverte pluridisciplinaire HAL, est destinée au dépôt et à la diffusion de documents scientifiques de niveau recherche, publiés ou non, émanant des établissements d'enseignement et de recherche français ou étrangers, des laboratoires publics ou privés. 
http://dx.doi.org/10.1016/j.epsl.2015.10.022

\section{GiantfossilmasswastingoffthecoastofWestlndia:}

\section{TheNatarajasubmarineslide}

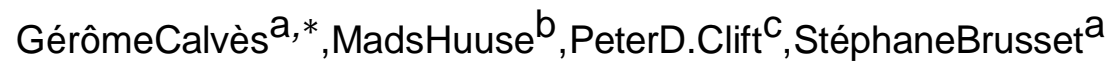

a

UniversitéToulouse3,PaulSabatier, GéosciencesEnvironnementToulouse, 14avenueEdouardBelin,31 400, Toulouse, France

*Correspondingauthor.

Emailaddress.gerome.calves@get.obsmip.fr(G.Calvès).

bSchoolofEarth,AtmosphericandEnvironmentalSciences, UniversityofManchester,ManchesterM139 $P L, U K$

C

DepartmentofGeologyandGeophysicsandCoastalStudiesInstitute, E235HoweRussell,LouisianaState University,BatonRouge, Louisiana70803,USA

\section{Abstract}

Weusetwodimensionalprestackdepthmigratedseismic reflectionprofilesandseafloor bathymetrytodescribe the continentalmarginstructureanda massivemasstransportdeposit off the westcoastofIndia.ThisgiantsliderunsfromtheGujurat-SaurashtramargintotheLaxmiBasin.Itisover330km long,amaximumof190kmwideanditsrunoutbasalgradientis $1.2^{\circ}$. Wenamethisgiantmasswastingdepositthe NatarajaSubmarineSlide.Thisslidecovers $49 \pm 16 \times 10^{3} \mathrm{~km}^{2}$ andrepresentsavolumeof $19 \times 10^{3} \pm 4 \times 10^{3}$ $\mathrm{km}^{3}$,makingitthesecondbyvolumeofanypassivemarginlandslide/masstransport deposit.Seismicfaciesanalysisallowstheinternalstructureofthemasstransportdeposittobedescribedasfaras thetoe.Thisslidehasbeenabletocircumventmassiveseamounts, thushighlightingthecapacityoftheflowandits potentialenergyduringemplacementinafunnelbetweentheslopeoftheWesternlndianpassivemarginandtheL axmiRidge.Stratigraphically,theemplacementoftheNatarajaSlidepredatesthemainpulseof sedimentation duringthe lateMiocene-Recentassociatedwith the IndusFanbutfollowsrapidsedimentationacrossSandSEAsiaduringtheEarlyMiddleMiocene.Themarginarchitectureattheheadofthisslideisassociatedwithagravitycontrolledfoldandthr ustbeltthatmayhavecausedslopesteepeningandtriggeringoftheslide. 


\section{Introduction}

Landslidesoccuronandoffshore, andrangein volumefromdm ${ }^{3}$ to $\times 10^{3} \mathrm{~km}^{3}$.

Triggeringmechanismsareoftenunknownandalmostcertainlydifferbetweensettings(Hamptonetal.,1996).Massw asting/masstransportdepositsrepresentsomeofthemostchallengingandimportantsedimentaryandmechani calfeaturesforgeoscientistsandstructuralengineerstounderstand(e.g.,Massonetal.,2006).Offshore,alongb othpassiveoractivemargins(MoscardelliandWood,2015), masswastingplaysanimportantroleinshapingmost continentalslopesthatleadtodeepabyssalplainsandhelpstoformthearchitectureofdeepwatersedimentarysy stemswhenassociatedwithturbiditycurrents(Embley,1976;Woodcock,1979;Bellaicheetal.,1986).Massmov ementscan,incertaincircumstances,affectmanmadeinstallations, suchasdeepseacablesorsubseaoffshore structures(Gennesseauxetal.,1980;Piperetal.,1988).Movementoftheoverlyingwatercolumnduringemplace mentmaygenerate

tsunamis, asdocumentedforthewellknownHoloceneStoreggaSlideintheNorthAtlanticoffshoreNorway(Jans enetal.,1987;Buggeetal.,1988;Yamadaetal.,2012).Despitethenumerousseafloorfeaturesdescribedandthe proposedmechanismsfortriggeringslides(e.g.,overpressure,fluid/sedimentremobilization,earthquake)theli mitednumberofgiantslidesintherockrecordthathavebeenstudiedindetaildonotallowadefinitiveanalogytobe madebetweenthesemodernandancientexamples.

ThemorphologyofthepresentdaymarginofwesternIndia(NainiandKolla,1982;Chakrabortyetal.,2006)isc haracterizedbyaseriesofarcuateconcaveupandconcavedownfeaturesattheshelftoslopetransition(Fig.1). Atthisscalewecandefinethemarginasbeingassociatedwithaseriesofprogradingsedimentarywedgesand/o rretrogradingerosiveslopes(Steffensetal.,2003). Themainsedimentarybodywithinthispartofthewesternlnd ianOceanisthelndusFan(Fig.1B)thatcontainsupto

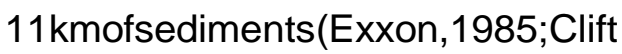

etal.,2001).Thesurficialshapesfoundalongthemargincanbesummarizedbythreedipbathymetricprofiles(Fig. 1C). ThenorthernprofilecorrespondstothelndusFanandshowsaconvexupwardprofilereflectingtheexcessof Plio-

Pleistocenesedimentsaccumulatedbythisthicksedimentarybody(Fig.1B).Althoughtheinfluenceofdeltaicse dimentarysystemsdiffersbetweenthethreeareas,wenotethattheprofilealongtheGujurat-

Saurashtrasectorshowsaconcaveupwardgeometrycomparedtoitsneighbors,suggestiveofmasswasting(Co lemanandPrior,1998;AdamsandSchlager,2000).SlopefailureofPlio-

Pleistocenedepositsspatiallyassociatedwithhydrates,rapidsedimentationratesorseismicityhasbeendocum entedalongtheslopeoftheWesternIndianmargin(Raoetal.,2002).

TheLaxmiandGujurat-

SaurashtraBasinshavebeenextensivelydescribedandanalyzedinrelationtotheriftinghistoryofGondwana,wit hparticularattentionhavingbeenpaidtothecontinentoceantransition(e.g.Bhattacharyaetal.,1994a;Todaland Edholm,1998;Chaubeyetal.,2002;Krishnaetal.,2006;Calvèsetal.,2008,2011;Misraetal.,2015).Nonetheless ,detaileddescriptionofthedriftsequencesoftheGujurat-

SaurashtraandLaxmiBasinshasnotbeenperformedpreviouslybecauseofthelimitedavailableseismicreflecti 
oncoverageandmodestnumberofwells/boreholesdrilledinthearea.Ourstudybuildsonpreviousstudiesandus estwo

dimensionalPreStackDepthMigrated(PreSDM)seismicreflectiondatatodescribethelargescalegeometryoft hemargin.Weidentifysomekeyfaciesthatformamajorsedimentvolumeandrepresenttheproductofamajorlate Mioceneeventinthehistoryof

thismargin.Wedefinethespatialextent,theinternalstructureandtectonicoriginofthispreviouslyundescribedse dimentarybody. ThisseismicallyobservedstructurehasrecentlybeencalibratedduringInternationalOceanDis coveryProgram(IODP)Expedition355(Pandeyetal.,2015).PreliminaryresultsfromSitesU1456andU1457(Fi g.1A), supportourinitialinterpretationsthatwerecompletedbeforedrilling. 

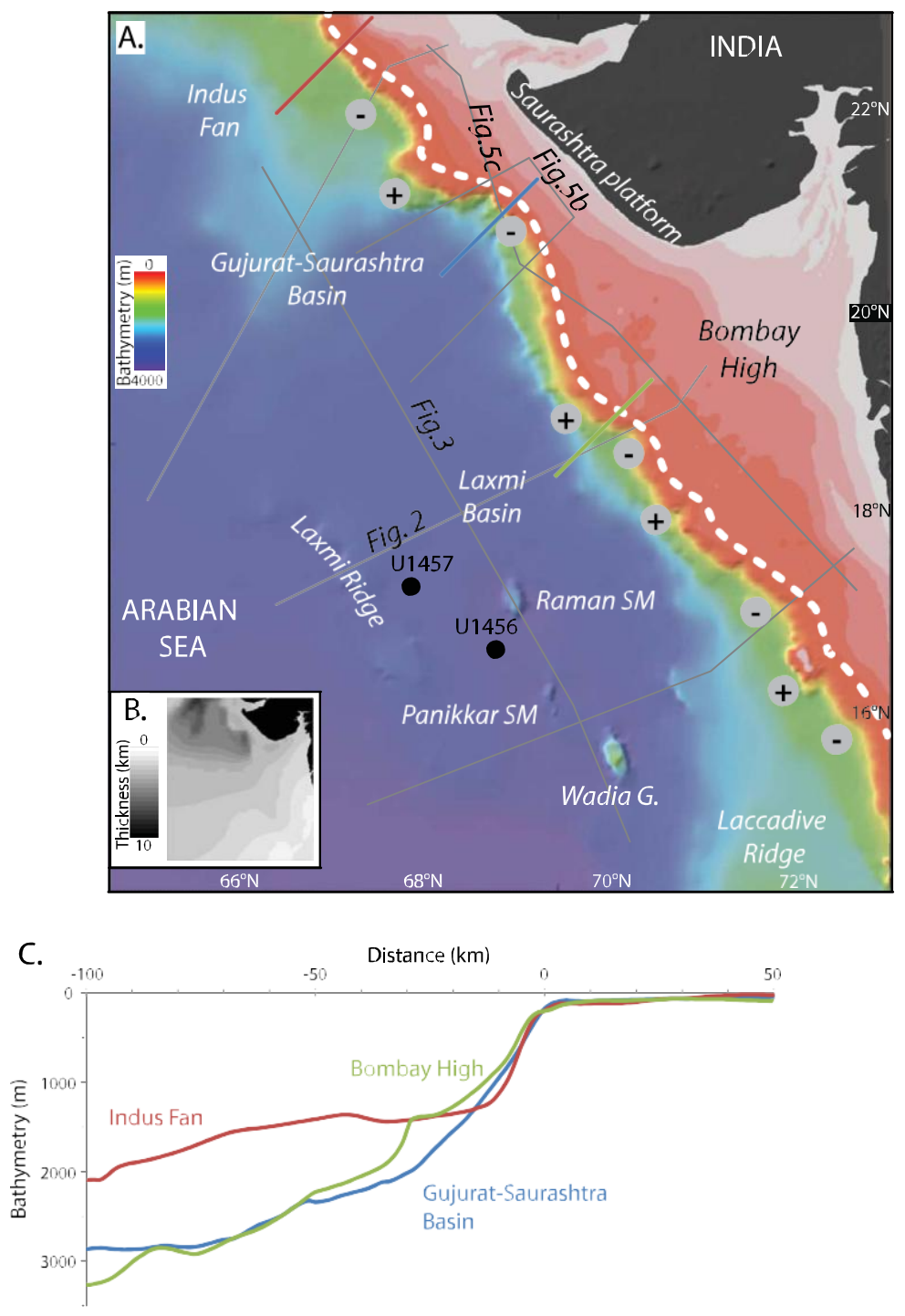

Fig.1.A.RegionalsettingoftheWesternIndiamarginandadjacentArabianSeaBasin. Theseismicdatause dforthisstudyaremarkedbygreylines.Alongmargin,convexupandconvexdownmorphologyofshelftoslo peareexpressedbypositiveandnegativegreysymbols, respectively.B.Thicknessmapofsedimentswithin thestudyarea(Exxon,1985), notetheextentofthelndusfandepocentrenorthofthestudyarea.C.Bathymet ricprofilesacrossshelfandslopefromthreelocationsalongthemargin,theseprofilesareeitherconvexup(I ndusFan,i.e.highsedimentrateandimportantdeepseafan)orconcaveup(BombayHighorGujuratSaurashtraBasin).ThetwolODPdrillsitesU1456andU1457arelocatedinthesouthwesternpartofthestud yare(Pandeyetal.,2015).

\section{Materialsandmethods}

Theapproximately3500kmofseismicreflectiondatausedinthisstudy(Fig.1A)arepartofthemuchlargerregi 
onallndiaSPAN ${ }^{\mathrm{TM}}$ surveyacquiredandprocessedbylONGXTechnologyin2006-

2007. Theacquisitionwasdesignedforcrustalscaleimagingbyusingalongreceivercablewitha $10.1 \mathrm{kmmaximu}$ moffsetandalargesourcesizeof7480cuin.Crustalscaleimagingisachievedbyan18stwowaytimerecord(equi valenttoaround40kmdepth). Theprestackdepthmigrateddataweremigrated(KirchhoffPreSDM)usingvelocit iesderivedfromiterativetomographicvelocitymodeling.VintagesinglechannelseismicreflectionprofileRC17 07fromtheLamont-DohertyEarthObservatoryhasalsobeenused(Fig.1;accessedbytheGeoMapApp3.3.9_ link:http://www.geomapapp.org/).

Weanalyzedtheseseismicreflectionlinesattwodifferentscales,fromthemega(basin)scaletothearchitecturalel ementscale.Atthescaleofthearchitecturalelements,seismicfaciesanalysis(Mitchumetal.,1977)wasusedtodocu mentanddescribethekinematicindicatorsofthemasstransportcomplexes(MTC)asdefinedbyBulletal.(2009).Ide ntificationoftheheadwall,extensionaldomain,translationaldomainandcontractionaltoedomains(Prioretal., 1984)i sbasedontheexternalandinternalgeometryofthemasswastingpackage,asimagedbytheseismicreflectiondata. $T$ heclassificationofattached/detachedMTCfollowstheschemeofMoscardelliandWood(2008). TheregionalGEBC O(BritishOceanographicDataCentre,2003)bathymetricdataareusedtodescribethemarginandlocatethevarious structureswheretheslideispresent.

Inspring2015,twolODPsitesweredrilledinthesouthwesternpartofourstudyarea(Fig.1A).Werefertothispr eliminaryagecontrolandsedimentaryfaciesfromonboardanalysistocalibrateourgeophysicalobservations $(P$ andeyetal.,2015).

\section{Results}

\subsection{Marginscalestructure-Natarajaslidelocation}

ThepresentdaystructureofthewesternIndianmarginismainlydominatedbyaseriesofinfilledriftbasinsspan ningfromthelandwardGujurat-

SaurastrahmargintotheLaxmiBasinandboundedtothewestbytheLaxmiRidge,whereseawarddippingreflect ors(SDR)markthetransitionbetweenabortedoceanicbasincrusttothetrueoceanicaccretiongeneratedalong theCarlsbergRidge(e.g.NainiandTalwani,1982;Calvèsetal.,2011;Misraetal.,2015).Thissettingistheresulto frepeatedridgejumpmarkedbySDRsequencesonbothsidesoftheLaxmiRidge.

ThemaingeodynamiceventtohaveshapedtheonshorepartofthemarginistheDeccanLargelgneousProvin ce(e.g.WellmanandMcElhinny, 1970;Mahoney,1988). Thiseventismarkedseawardbyaprominentseismicre flectionpackageoverlainbycarbonateplatforms(Calvèsetal.,2008,2011)andinturnburiedbyCenozoicsilicicl asticdepositslargelysourcedfromthelndiancontinentandHimalayaviathelndusRiver(e.g.,Cliftetal.,2001)(Fi g.2A). TheflatlyingfloodbasaltsareintransitionwithseawarddippingreflectionpackagesintheLaxmiBasin(Calvès, 2009;Misraetal.,2015;Fig.2A). 

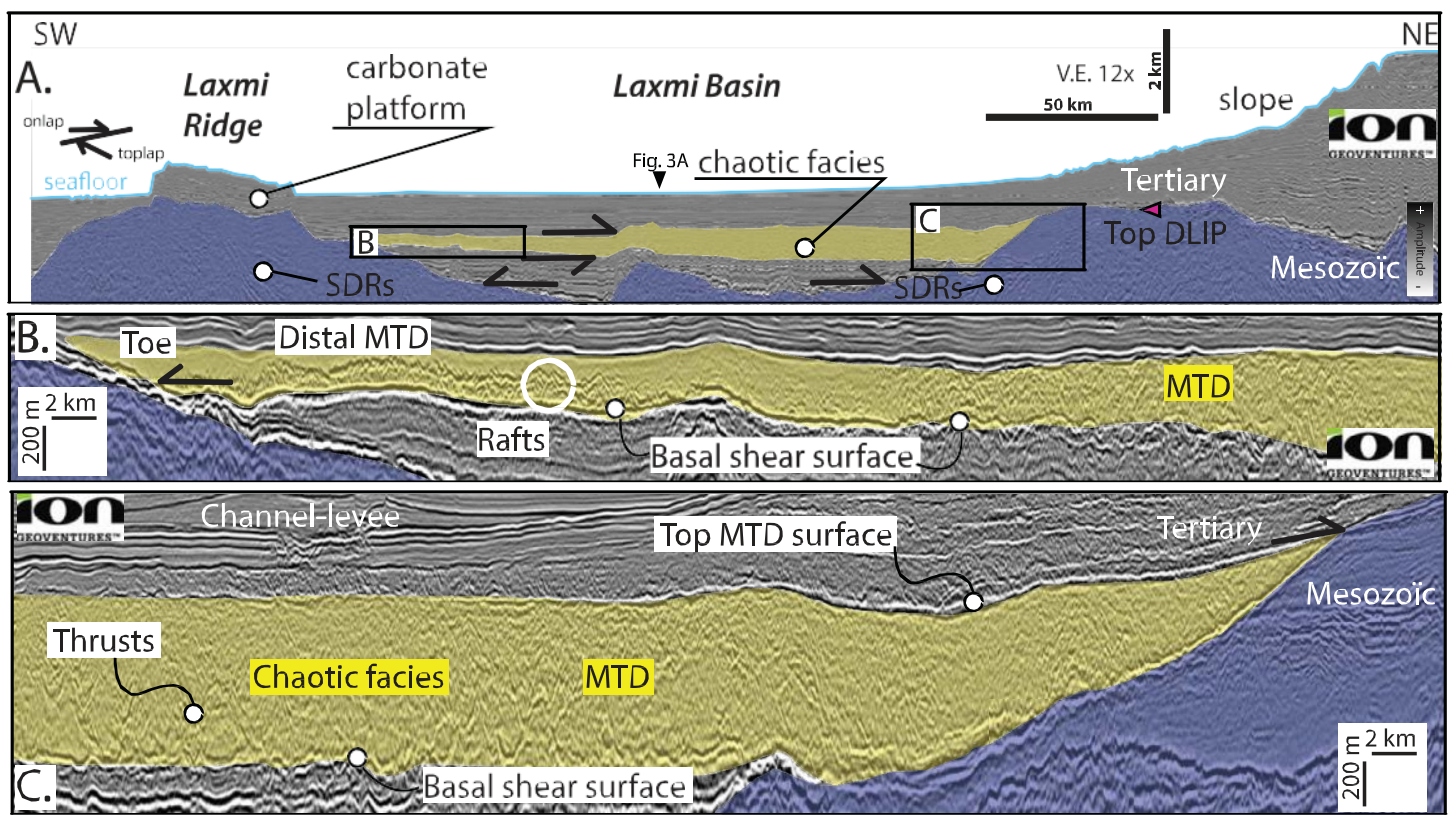

Fig.2. RegionalprestackdepthmigratedseismicreflectionprofilesacrosstheBombayHigh,LaxmiBasint oLaxmiRidge.A.Thisseismicreflectionprofileofthemarginshowsthewidthofanextensivechaoticseismi cfacieswithintheCenozoicsection.TheMeso-

Cenozoictransitionbelowtheslopeofthemarginismarked(purplefiledarrow)bythetopoftheDeccanLarg elgneousProvince(topDLIP)seismichighamplitudereflectiveevent.SeawardDippingReflector(SDR)s equencesareobservedonbothsidesoftheLaxmiBasin.B.Thechaoticseismicfaciesismarkedbymassiv ethrustandonlaplandwardontheMesozoicsequence.C.TowardtheLaxmiRidgethechaoticseismicfaci esthinslaterally.D.TowardthePanikkarSeamountthefaciesislesschaoticandtheslidethinsandonlapsa gainstthestructuralbasementhighs,seeFig.1Aforprofilelocation. 

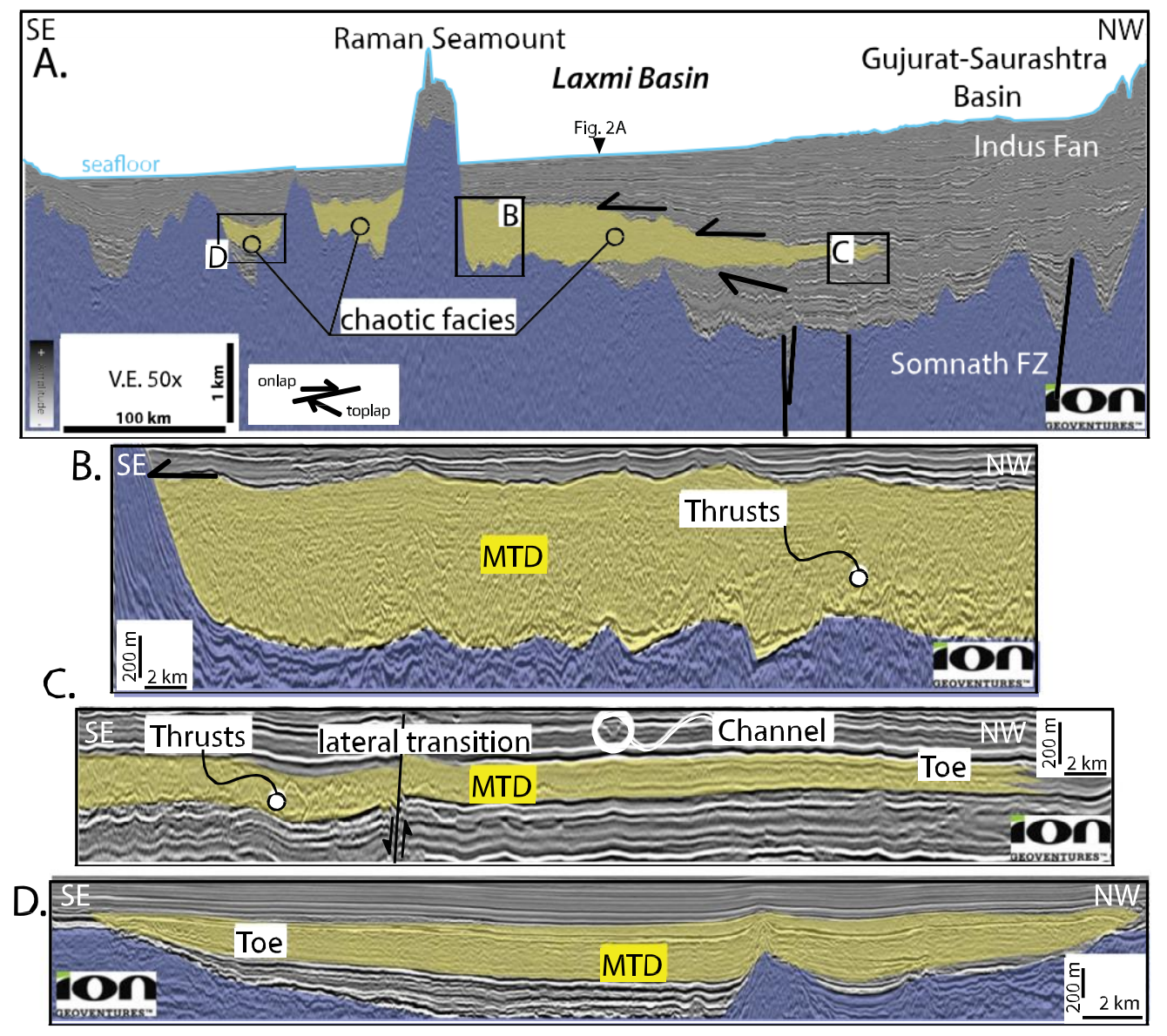

Fig.3.RegionalprestackdepthmigratedseismicreflectionprofilesNW-SEacrossthe LaxmiBasinand RamanSeamount and, Gujurat-

SaurashtratoLaxmibasinsB.,thechaoticseismicfaciesisthickertowardtheSW, notetheportionofthelnd usFananditsgiantchannellevees. Thechaoticseismicfaciesrunsfurthersouth,beyondtheRamanSeam ount.Notechangeofscalebetweendisplays,seeFig.1Aforprofilelocation. 


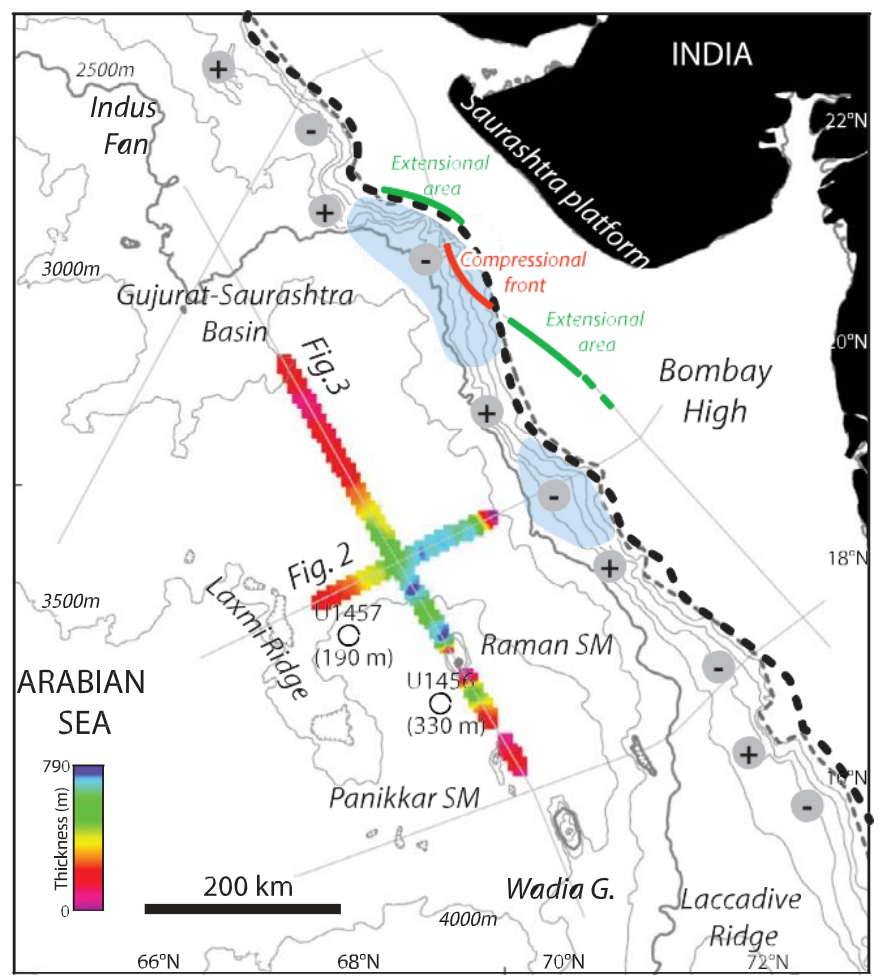

Fig.4.IsopachmapoftheNatarajaslide. Theseafloorcontoursarespacedevery500meters. Thegeomorp hologicalelementsoftheseafloorsurfacearesimilartoFig.1.Thicknessofthemasswasteddepositsofthet wodrillIODPsiteU1456andU1457aremarkedinbrackets(Pandeyetal.,2015).Twoconcavedownmorpho logiesofthemarginaremarkedintheslopebyblueshadedareas. Thesearemarkingpotentialmassmissing totheirlateralsectionalongthedownslopeoftheWestlndianmargin. Theshelfmargingeometryisrelatedtol argescalegravityspreadingoftheslopewithconcavedownareasunderlainbycontractionaldeformation(s eeFig.5).(Forinterpretationofthereferencestocolorinthisfigure,thereaderisreferredtothewebversionoft hisarticle.)

TheCenozoiclndusFansequenceiscomposedatitsbasebycontinuousparallelseismicreflectionsthatonlap ontostructuralhighs(Figs.2B,2C,and3A).Abovethissequenceapackagedefinedbychaoticfaciesofregionalex tentcoversmostoftheLaxmiBasin. ThispackagethinstowardsandeventuallyonlapstheLaxmiRidgetotheSE(Fi g.2A).ItisthickertowardstheNE, whereitis $>750$ mthick(Fig.4). Theinternalorganizationofthissequenceiscomp osedofchaotictodiscontinuousseismicfaciespackages,brightsteeplydippingreflections, rotateddiscontinuou sstratalreflectionsandlowamplitudestructurelessfacies(insetsFigs.2Aand2B). Eachofthesefaciescanbeinte rpretedasmasstransportdepositsandhelpustodefinethepotentiallocationoftheultimateheadscarpandtoe(e. g.,Bulletal.,2009)(Figs.2,3,4and5).The2Dgriddoesnotallowcompletemappingofthemasstransportdeposit,b uttheoverallextentandlargescalethicknessvariation, andthusvolume, isconstrainedwithreasonablecertainty. Theseismicreflectionprofilesinthedeepbasinallowonlytheidentificationofthetoeregionoftheslide(Figs.2B,3 C,and3D).Weareabletodefinetheedgeoftheseawardpartoftheslidewherethindepositsandlesschaoticfacies( 
Figs.2B,3C,and3D)areexpressedtotheNW,SWandSE(Fig.4).TheNEtoSpolarityoftheslideandespeciallyitst oehasbeendrilledatbothIODPSitesU1456andU1457(Pandeyetal.,2015).Regionalslopestothebasement,a swellasthemodernbathymetryarealsoconsistentwithemplacementtowardsthesouth. Thechaoticfaciesonlap sthefootoftheRamanSeamount(Figs.3Aand3B).TheRamanandPannikarSeamounts,locatedinLaxmiBasin (Figs.1Aand3B;Bhattacharyaetal.,1994b),constitutedbarrierstotheflowoftheslidedeposit,whichflowedarou ndthesefeaturestowardsthesouth(Fig.3B). Thecoredmasstransportdeposit(Fig.4)thicknessvariesfrom 33 Om atSiteU1456to 190m

atSiteU145(Pandeyetal.,2015).ThesethicknessvariationsintheSWpartoftheNatarajaslideareinagreement withtheisopachmapcomputedfromthetopandbaseoftheseismicallydefinedchaoticfaciesinthisregion(Fig.4).

ThearchitectureoftheproximalpartofthemarginoffshoretheSaurashtraplatform(Figs.3,5Band5C)iscomp osedofadeepwaterfoldandthrustbeltthatinvolvestheCenozoicsedimentarypackage.TheNWpartofthestud yareashowsathinsedimentarycover,comparedtotheadjacentareas, withatransitionintoamajortroughwhere twoburiedstackedcompressionalfeaturesareidentifiedandwhichareseentoemanatefromdetachmentsthatr unintotheslopeofthemargin. Thesetectonicstructuresarelinkedtoextensionalstructuresthatseesignificantpi lesofsedimentextendingtowardtheSE(Fig.5C). Themaindepocenteroffthewesternmarginoflndiaisassociat edwiththeBombayHigh(e.g.,Biswas,1982;Rao,1991;Figs.1Band5A).

TheheadwalldomainofthismasswastingcomplexislocatedintheslopeoftheGujuratSaurashtraBasin.Basedonthemorphologyofthemargin,twoconcaveupareasarelocatedupslopeoftheNatar ajaSlide(blueshadedareas,Fig.4).BasedonthepolarityoftheseismicfaciesanddrillingresultsfromIODPExpe dition355, wefavorthenorthernpolygon(northof20 N,Fig.4)aspotentialheadwallsourceforthefollowingreaso $\mathrm{ns}$ :Basedonbathymetry/slopegradientandseismicprofiles, theheadwallisinferredtorunalongtheslopeandsh elfbreak(Figs.1and5A). Thetruncatedreflectionsattheshelfedge/slopetransitionmarkstheheadofthescar(Fi g.5B).Slopegradientsintheheadscarpregionrangefrom $1.6^{\circ}$ to2. $7^{\circ}$, whicharetypicalofthedécollementlevelin upperslopeandheadscarpregionsalongpassivemargins(HarrisandWhiteway,2011).Thedistalbasallowerh alfgradientofthemasstransportdepositsliesatagradientof $1.2^{\circ}$. Thisvalueisconsistentwiththelargestslidesth attendtooccuronthelowestsurfaceslopes(McAdooetal.,2000).

BeforetherecentdrillingofSitesU1456andU1457, therewasnopreciseageinformationforthelandslidefacie sitself.However, itsageisbracketeddownwardbythepresenceofupto1150mofbasinfilldepositbetweenthesli deandthetopofthevolcanicbasementandoverlyingPaleogenecarbonateplatforms(Calvèsetal.,2008), andu pwardbythechannelleveecomplexesofthelndusFan,whicharedatedasEarlyMioceneandyoungerfurtherno rth(Cliftetal.,2001). Thestratigraphicpositionofthemasstransportdepositsiscalibratedfurthernorthwhereitst opandbasemarkerscorrelatewithhemipelagicsedimentsfromaboreholewithpoorlyresolvedagesfromearlytol ateMiocene( 15Ma)(http://www.dghindia.org;well:GSDW11A)(Figs.2Aand3A).AtSiteU1456(Fig.1A),thesedi mentlyingimmediatelyaboveandwithinthemasstransportdepositisdatedat< $10.8 \mathrm{Ma}$, whereassedimentlyingdire ctlyunderneaththedepositis datedas13.5-

17.7Ma(Pandeyetal.,2015).TheagegapbetweenthetworepresentserosionatthedrillsitelinkedtoMTCempla cementaround10.8Maanddoesnotindicateslowsedimenttransport. ThebestageestimatefortheNatarajaslid eisthusbetweenaround10.8Ma(lateMiocene). 


\subsection{SizeandvolumetricsoftheNatarajaMTD}

ThesizeoftheheadscarpintheGujurat-SaurashtraBasinis 100kmalongtheNWSEaxisand 90kmalongitsNE-

SWaxis(Figs.4and5A). Itspresentdayminimumheightis $2300 \mathrm{~m}$, thiscorrespondstothetopofthetruncatedr eflectionsontheshelfedgetransitiontothebaseoftheslopewherecontinuousreflectionbegins(Fig.5B). Thee stimatedvolumeoftheheadscarpcanbecomputedasthequarterofanellipsoid,sothatitsvolumeis

estimatedat $\sim 11 \times 10^{3} \mathrm{~km}^{3}$. TheNatarajaSlidehasa surfaceextentof $49 \pm 16 \times 10^{3} \mathrm{~km}^{2}$ and a volumeofsome $19 \times 10^{3} \pm 4 \times 10^{3} \mathrm{~km}^{3}$, whichmakesthis oneofthelargestknownmasstransportcomplexdepositsalonganypassivemarginsofardocumentedonEart h.Atthescaleofthemargin,thesliderepresents $8 \% \pm 3 \%$ oftheCenozoicsedimentdepositedintheGujuratSaurastrahandLaxmiBasins(Fig.1B; Exxon,1985, wholevolumeof $600 \times 10^{3} \mathrm{~km}^{3}$ ). Theminimumvolumeof thescarpobservedonthebathymetryandtheestimatesoftheNatarajaSlidedepositareofthesameorder.Thea realsize $>100 \mathrm{~km}^{2}$ andvolume $>1 \mathrm{~km}^{3}$ oftheNatarajaSlideallowustoclassifyitasanattachedmasstransportd epositintheterminologyofMoscardelliandWood(2008,2015). 


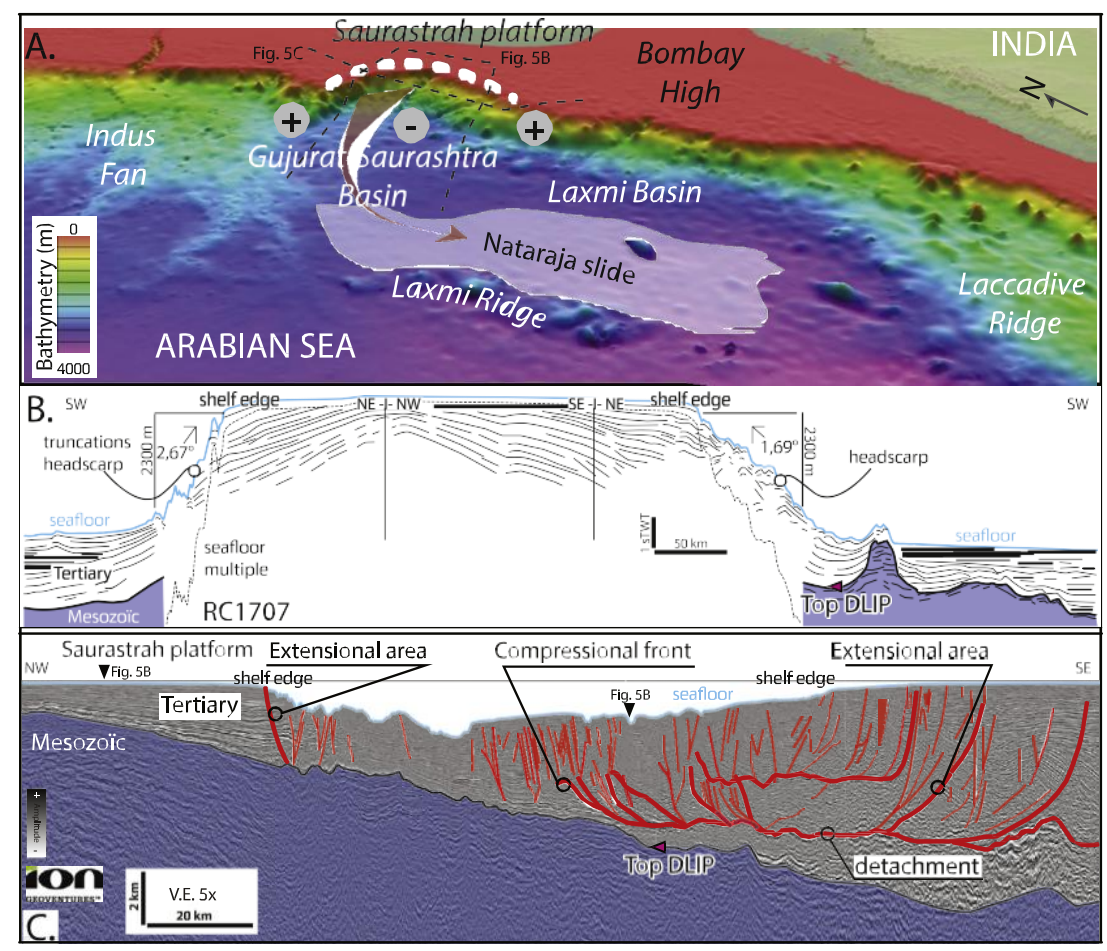

Fig.5.A.ExtensionoftheburiedNatarajaslideandpotentialsourcearea.Therunoutdistanceisabout550k mforalengthof $338 \mathrm{kmandamaximumwidthof} 193 \mathrm{~km}$, volumetricestimatesis $19 \times 10^{3} \pm 4 \times 10^{3} \mathrm{~km}^{3}$. B. Lin edrawingofseismicsinglechanneIRC1707(Columbia-LamontDohertyEarthObservatory,GeoMapApp).C.RegionalNWSEprestackdepthmigratedseismicreflectionprofileacrosstheshelfandslopeoftheWestIndiacontinenta ImarginoffshoretheSaurashtraplatform. Thestructural styleoftheTertiarysectionisassociatedwithastackoftwocompressionaldeepwaterfoldandtrustbelt.De ccanLargelgneousProvinceismarkedwithapurplearrow(topDLIP). 


\section{Table1}

Volumetricandheightoffallhorizontalrunoutratioofthe'giant'submarinelandslides(i.e.volume $>10^{3} \mathrm{~km}{ }^{3}$ ) andassociatedreferences.

\begin{tabular}{|c|c|c|c|c|c|}
\hline Slidename & Runoutlengt & Height $H$ & H/Iratio & Vol & Reference \\
\hline 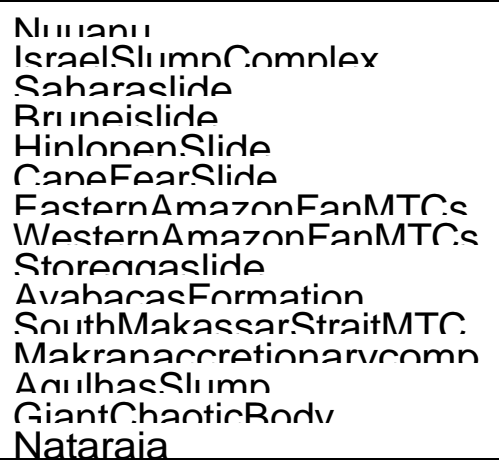 & 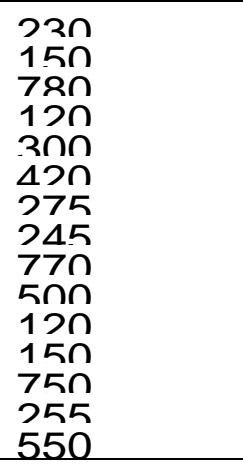 & 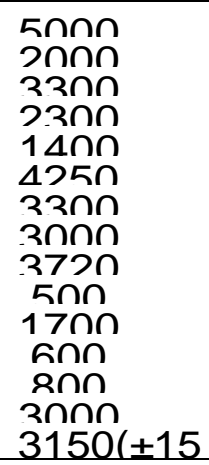 & 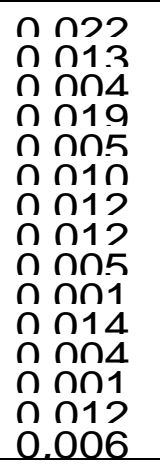 & 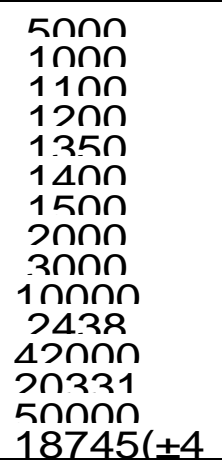 & 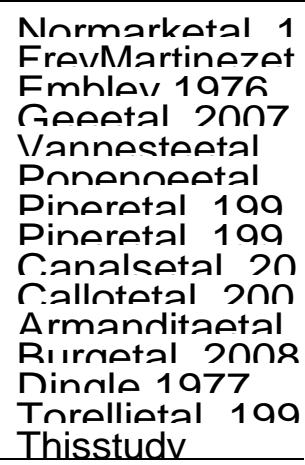 \\
\hline
\end{tabular}

\section{Discussion}

\subsection{SignificanceoftheNatarajaSlide}

Wehavecompiledfromvarioussourcestheheightoftheratiooftheoriginatingslopeheighttohorizontalrunoutofsubm arineslidesasafunctionofthevolumeofeachmasswastingdeposit(e.g.,EdgersandKarlsrud,1982;DeBlasioetal.,20 06).Globally,theNatarajaSlideisthesecondlargestmarinemasstransportdepositalongapassivemarginbyvolume, onlysmallerthantheAgulhasslump(Dingle,1977)(Table1,Fig.6).Basedonthesizeandshapeofthisbody,thelongrun outofthismasstransportdepositacross a gentleslope,coveringlongdistancesandthedevelopmentofathickdepositfurtherpushestheenvelopeproposedby DeBlasioetal.(2006), aswellastheempiricalcurvesofsubmarinelandslidesofEdgersandKarlsrud(1982)totheirli mits. Thisslidehasasourcelocatedinamixedsedimentarymarginsequenceanditssinkisstructurallyboundedb ymajorbathymetricfeatures,whichwearguehavefacilitateditsrunoutdistancebyfocusingit. Thepotentialmixed lithologiessourcedfromtheslopeoftheWestIndianMargincouldexplainthevariousfeaturesobservedwithinthe coredslidedeposits(Pandeyetal.,2015).TheobservedolistostromesintheMakran(onshorePakistan,Burgetal .,2008)ortheGiantChaoticBody(offshoreGibraltar,Torellietal.,1997)arethelargestchaoticsedimentarybodies yetobserved. Thesetwosedimentarybodiesshareaspecificgeodynamicandstructuralsetting, anaccretionary prism, whichisdifferentfromthepassivemarginstructuralframeworkoftheWestlndianMarginwhereweobserve theNatarajaSlide(e.g.NainiandTalwani,1982). 
Atthemarginscale,thestratigraphicandstructuralsettingoftheproximalsourceareaallowsustoproposeaspa tialassociationoftheheadwallandthecompressionalfrontbelowtheslopeofthemargin(Fig.5C). Thisconfigurati on,whichisfoundalongmanypassivemargins, accretionaryprismsandtransformmargins, isasourceofmanysli desandofmasswastinggenesis(e.g.,Morley,2009;Morleyetal.,2011).Atthescaleoftheslidethekinematiceviden cefora

singleeventassociatedwiththisslideisrelatedtothegeometryofthecompressional/extensionalfeaturesfromitsbodyi ntoitstoe.Theabsenceofmultiplestackedcompressionalfeaturesin this slide(Figs.2B,2Cand3B)arguefor a singlemassmovement. A

basalshearsurfaceisonlyidentifiedatthebottomofthemasstransportcomplex(Fig.2B).

ThepotentialperiodofemplacementoftheNatarajaSlideiscoincidentwithmajorchangesinsouthernAsia(Clift, 2006).Onefactoristheclimaticevolutionandchangeinsedimentaryproductionandsedimentationaroundthecontine ntalmarginsofSandSEAsiaatthattime.DuringtheearlyPaleogenethemarginrecordedcarbonateplatformgrowthfoll owedby

astressshiftexpressedbyfocalclasticsedimentaryloading(Whitingetal.,1994)thathasplayedanimportantroleonbui Idingsequencesofsedimentarygravitationalsliding.ThisisobservedonothermarginsaroundthelndianOceansucha stheKrishna-

GodavariBasin(Rao,2001)andRovumaBasin(MahanjaneandFranke,2014).TheMiddletoEarlyMiocenewasatim eofrapidsedimentdeliverytothecontinentalmarginsofIndia,drivenbyfasterosioncausedbyaheavysummermonsoo n.Developmentofa

majorclasticwedgemayhaveresultedingravitationalinstabilitythatresultedincollapse 10.8Ma.
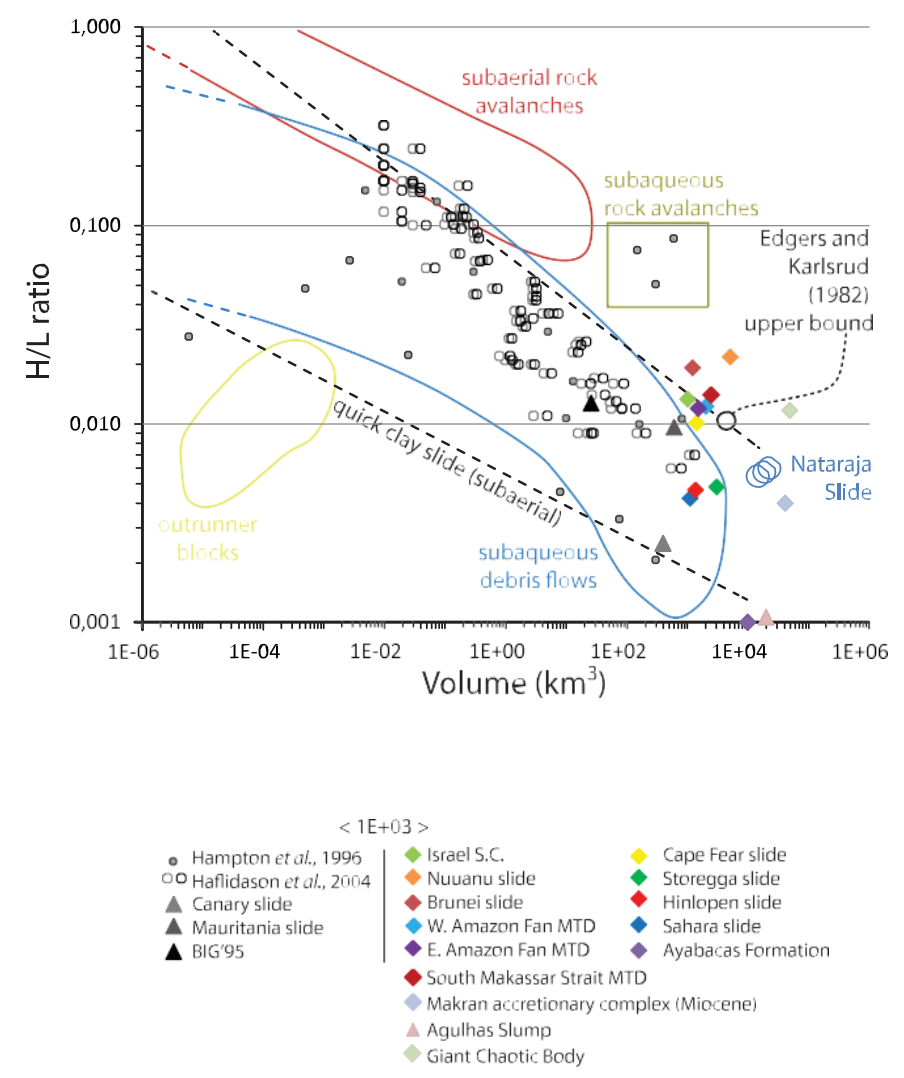
Fig.6. Heightoffalltohorizontalrunoutratioofthe slide as a functionof thevolumeofthemasswastingdeposits. ThecoloredenvelopesarefromDeBlasioetal.(2006,seewithinfor compileddata).IndividualslidesarefromHaflidasonetal.(2004)fortheStoreggaslideoffNorwayandfromv ariousslidesintheglobalocean(Hamptonetal.,1996).Giantslidesaredepictedbytrianglesorcoloreddiam ondsforvolumes $>10^{3} \mathrm{~km}^{3}$ (seeTable1 forreferences).CurvesfromEdgersandKarlsrud(1982), theupper boundvaluesforsubmarinelandslidesandsubaerialquickclayslidesareplottedforreference.(Forinterpre tationofthereferencestocolorinthisfigurelegend,thereaderisreferredtothewebversionofthisarticle.)

\subsection{Perspectives}

Regionally,theNatarajaSlide,ifassociatedwithasinglecatastrophicevent,couldhaveleftitsmarkonthecoas talrecordoftheArabianSeaintheformoftsunamigenicdeposits,althoughthe >10.8Maageofthedepositmaym akepreservationanissuewhensearchingforequivalentsedimentsonshore. Thestructuralanalysisofdensergr idofdataontheslideshouldallowdeterminationofthekinematicsofthisslideandthepotentialmechanismsofitse mplacement(catastrophicorcreeping). ThelongrunoutandtherelationshipwiththeseamountsintheLaxmiBa sinhowevercouldarguesfromcatastrophicemplacement.Specificcharacterizationofthesedimentsassociat edwiththeslideisneededtoinferanyroleplayedbybaselevelvariation,hydratedissociation,localtectonicsorev enbasinfluidmigrationinrelationwithhydrocarbongeneration.Comingafteratimeofrapidmarginsedimentatio nmayargueinfavorofgravitationalinstabilityastheunderlyingcause.

IntheArabianSeathepresentdayriskofcoastaltsunamisisanalyzedassumingasourceofmassmovementsf romtheMakranaccretionaryprism,oreventheAndamantoJavaarcinthecaseoffarfieldsources(e.g.Heidarza dehetal.,2008;OkalandSynolakis,2008).Thisapproachisassociatedwiththelimitedoccurrenceofearthquak esalongthewesterncontinentalmargin(Chandra,1977), becausethesearecommoncausesoftsunamisinthel ndianOceanregion.Yetnoriskassessmentisdiscussedinthesestudieswithasourceofmassmovementsandw atercolumndisplacementsrelatedtotheWestIndianContinentalmargin.OurdescriptionoftheNatarajaSlidehi ghlightsthisregionasapotentialsourceofmassmovementsintheWesternIndianOceanandshouldguidefutur eresearchonthismargin.Giventhelargesedimentmassthathasbeenbuiltalongthewesterncontinentalmargi nduringthePlio-

Pleistocenephaseofrapidsedimentdeliverythepotentialforfurthermajormasswastingeventsmaywellexist. T hisnewinformationaboutalargeLateMioceneeventiscentraltorefiningourassessmentoftsunamihazardalon gthedenselypopulatedwesterncoastofIndia.Convexupwardareasalongthemarginaremorelikelytoslip,thisri skisunderlinedbythedepthandslopeofthedécollementobservedbelowtheSaurashtrashelfthat isdeeperthanitformedoriginally.

\section{Conclusions}

ThispaperreportsthediscoveryandfirstdescriptionandquantificationofthegiantNatarajaSlideoffshoreMu mbai,thesecondlargestlandslidesofardiscoveredalonganypassivemarginworldwide.Thelandslidecoversar ound $5 \times 10^{4} \mathrm{~km}^{2}$ andcomprisessome $2 \times 10^{4} \mathrm{~km}^{3}$ sediment. ItislateMioceneinage. TheNatarajaSlide'sroleinf 
ormingatopographicalhighfortheeasternborderofthelndusFancanbe

questioned.Inbothactiveandpassivemarginsweshouldconsiderthatmasswastinghasimportancealongside carbonateaccumulationandfluvialinputinshapingtheseafloorofsedimentarybasins. TheNatarajaSlidewillre quirefurtherstudiestofullyunderstanditsstructureandspatialorganization, whichmaybeachievedinpartwithf utureworkonthecoresrecoveredbyIODPExpedition355.

\section{Author contributions}

G.C.hasframedthestudyoftheNatarajaSlide,andallauthorshavecontributedtothemainideadescribedinthi smanuscriptandtoitswriting.

\section{Acknowledgements}

TheauthorswouldliketothankIONGeoventures ${ }^{\circledR}$ forallowingtheuseofthelndianSpan@seismicsurveyandp ublicationofthiswork. WearealsogratefultolHS ${ }^{\circledR}$ foruseofKingdomsoftwareandMidlandValley ${ }^{\circledR}$ foruseofMov e.Wewouldliketothank

D.J.W.Piperforreviewofanearlierversionofthemanuscriptandinsightfulcomments. TheeditorPeterSheareran dreviewersJoeCartwrightandMichaelStrasserandananonymousreviewerarethankedfortheirconstructiveco mments.

\section{References}

Adams,E.W.,Schlager,W.,2000.Basictypesofsubmarineslopecurvature.J.Sediment. Res.70,814828.

Armandita,C.,Morley,C.K.,Rowell,P.,2015.Origin,structuralgeometry,anddevelopmentofagiantcoher entslide:theSouthMakassarStraitmasstransportcomplex.Geosphere11,376-403.

Bellaiche,G., Coutellier,V.,Droz,L.,1986.Seismicevidenceofwidespreadmasstransportdepositsinthe Rhônedeepseafan: their rolein the fanconstruction.Mar.Geol.71(3-4),327-340.

Bhattacharya,G.C.,Chaubey,A.K.,Murty,G.P.S.,Srinivas,K.,Sarma,K.V.L.N.S.,Subrahmanyam,V.,Kri shna,K.S.,1994a.Evidenceforseafloorspreadingin theLaxmiBasin,northeasternArabianSea.EarthPlanet.Sci.Lett.125(1-4),211-220.

Bhattacharya,G.C.,Murty,G.P.S.,Srinivas,K.,Chaubey,A.K.,Sudhakar,T.,Nair,R.R.,1994b.Swathbath ymetricinvestigationoftheseamountslocatedintheLaxmiBasin,EasternArabianSea.Mar.Geod.17(3) ,169-182.

Biswas,S.K., 1982.RiftbasinsinwesternmarginofIndiaandtheirhydrocarbonprospectswithspecialrefere ncetoKutchBasin.Am.Assoc.Pet.Geol.Bull.66(10), 1497-1513.

BritishOceanographicDataCentre,2003.GEBCODigitalAtlas,CentenaryEdition[CDROM],Br.Oceanogr.Da taCent., Liverpool,U.K.

Bugge,T.,Belderson,R.H.,Kenyon,N.H.,1988.TheStoreggaSlide.Philos.Trans.R.Soc.Lond.Ser.A,Mat 
h.Phys.Sci.325(1586),357-388.

Bull,S.,Cartwright,J.,Huuse,M.,2009.Areviewofkinematicindicatorsfrommasstransportcomplexesusi ng3Dseismicdata.Mar.Pet.Geol.26(7),1132-1151.

Burg,J.P.,Bernoulli,D.,Smit,J.,Dolati,A.,Bahroudi,A.,2008.Agiantcatastrophicmudanddebrisflowinthe MioceneMakran.TerraNova20,188-193.

Callot,P.,Sempere,T.,Odonne,F.,Robert,E.,2008.Giantsubmarinecollapseofacarbonateplatformatth eTuronian-Coniaciantransition:theAyabacasFormation,southernPeru.BasinRes.20(3),333-357.

Calvès,G.,2009.TectonostratigraphicandclimaticrecordoftheArabianSea.Ph.D.thesis.Univ.ofAberde en,Aberdeen,UK.19March,292pp..

Calvès,G.,Clift,P.D.,Inam,A.,2008.AnomaloussubsidenceontheriftedvolcanicmarginofPakistan:noin fluencefromDeccanplume.EarthPlanet.Sci.Lett.272(1),231-239.

Calvès,G.,Schwab,A.M.,Huuse,M.,Clift,P.D.,Gaina,C.,Jolley,D., Tabrez,A.R.,Inam,A.,2011.Seismicv olcanostratigraphyofthewesternIndianriftedmargin:thepreDeccanigneousprovince.J.Geophys.Res $.116, \mathrm{~B} 01101$.

Canals,M.,Lastras,G.,Urgeles,R.,Casamor,J.L.,Mienert,J.,Cattaneo,A.,DeBatist,M.,Haflidason,H.,Imbo, Y.,Laberg,J.S.,Locat,J.,Long,D.,Longva,O.,Masson,D.G.,Sultan,N.,Trincardi,F.,Bryn,P.,2004.Slopefail uredynamicsandimpactsfromseafloorandshallowsubseafloorgeophysicaldata:casestudiesfromtheCOS TAproject.Original ResearchArticle.Mar.Geol.213(1-4),9-72.

Chakraborty,B.,Mukhopadhyay,R.,Jauhari,P.,Mahale,V.,Shashikumar,K.,Rajesh,M.,2006.Finescaleanal ysisofshelfslopephysiographyacrossthewesterncontinentalmarginofIndia. Geo Mar.Lett.26 (2),114119.

Chandra,U.,1977.Earthquakesofpeninsularlndiaaseismotectonicstudy.Bull.Seismol.Soc.Am.67(5),1387-1413.

Chaubey,A.K.,GopalaRao,D.,Srinivas,K.,Ramprasad,T.,Ramana,M.V.,Subrahmanyam,V.,2002.An alysesofmultichannelseismicreflection,gravityandmagneticdataalong aregionalprofileacrossthecentral-westerncontinentalmarginofIndia.Mar.Geol.182(3-4),303-323. Clift,P.D.,2006.ControlsontheerosionofCenozoicAsiaandthefluxofclasticsedimenttotheocean. Earth Planet.Sci.Lett.241(3-4),571-580.

Clift,P.D.,Shimizu,N.,Layne,G.D.,Blusztajn,J.S.,Gaedicke,C.,Schlüter,H.U.,Clark,M.K.,Amjad,S.,20 01.DevelopmentofthelndusFananditssignificancefortheerosionalhistoryoftheWesternHimalayaan dKarakoram.Geol.Soc.Am.Bull.113(8),1039-1051.

Coleman,J.M.,Prior,D.B.,1998.Masswastingoncontinentalmargins.Annu.Rev. EarthPlanet.Sci.16,101-119.

DeBlasio,F.V.,Elverhøi,A.,Engvik,L.E.,Issler,D.,Gauer,P.,Harbitz,C.,2006.Understandingthehighmo bilityofsubaqueousdebrisflows.NorwegianJ.Geol.86,275-284.

Dingle,R.V.,1977.Theanatomyofalargesubmarineslumponashearedcontinentalmargin(SEAfrica).J. Geol.Soc.134,293-310.

Edgers,L.,Karlsrud,K.,1982.Soilflowsgeneratedbysubmarineslides:casestudiesandconsequences. 
Norw.Geotech.Inst.Bull.143,1-11.

Embley,R.W.,1976.Newevidenceforoccurrenceofdebrisflowdepositsinthedeepsea.Geology4,371374.

Exxon,1985.ExxonWorldMappingProject.TectonicMapoftheWorld.ExxonProductionResearchComp any.Houston,USA.

FreyMartinez,J.,Cartwright,J.,Hall,B.,2005.3Dseismicinterpretationofslumpcomplexes:examplesfro mthecontinentalmarginoflsrael.BasinRes.17(1),83-108.

Gee,M.J.R.,Uy,H.S.,Warren,J.,Morley,C.K.,Lambiase,J.J.,2007.TheBruneislide:agiantsubmarinela ndslideontheNorthWestBorneoMarginrevealedby3Dseismicdata.Mar.Geol.246(1),9-23.

Gennesseaux,M.,Mauffret,A.,Pautot,G.,1980.Lesglissementssousmarinsdelapentecontinentaleniç oiseetlarupturedecâblesenmerLigure(Méditerranéeoccidentale).C.R.Acad.Sci.Paris290,959962.

Haflidason,H.,Sejrup,H.P.,Nygård,A.,Mienert,J.,Bryn,P.,Lien,R.,Forsberg,C.F.,Berg,K.,Masson,D., 2004. TheStoreggaSlide:architecture,geometryandslidedevelopment.Mar.Geol.213(1-4),201234.

Hampton,M.A.,Lee,H.J.,Locat,J.,1996.Submarinelandslides.Rev.Geophys.34(1),33-59.

Harris,P.T.,Whiteway,T.,2011.Globaldistributionoflargesubmarinecanyons:geomorphicdifferencesb etweenactiveandpassivecontinentalmargins.Mar.Geol. 285 (1-4),69-86.

Heidarzadeh,M.,Pirooz,M.D.,Zaker,N.H.,Yalciner,A.C.,Mokhtari,M.,Esmaeily,A.,2008.Historicaltsunamii ntheMakranSubductionZoneoffthesoutherncoastsoflranandPakistanandresultsofnumericalmodeling. OceanEng.35(8-9),774-786.

Jansen,E.,Befring,S.,Bugge,T.,Eidvin,T.,Holtedahl,H.,Sejrup,H.P.,1987.Largesubmarineslidesonthe Norwegiancontinentalmargin:sediments, transportand timing. Mar.Geol. 78(1-2),77-107.

Krishna,K.S.,Rao,D.G.,Sar,D.,2006.Natureofthe crustin the LaxmiBasin(14$20^{\circ} \mathrm{N}$ ), westerncontinentalmarginofIndia.Tectonics25(1),TC1006.

Mahanjane, E.S., Franke,D.,2014. The

RovumaDeltadeepwaterfoldandthrustbelt, offshoreMozambique.Tectonophysics614,91-99.

Mahoney,J.J.,1988.Deccantraps.In:Macdougall,J.D.(Ed.),FloodBasalts.Kluwer,Dordrecht,pp.151-191.

Masson,D.G.,Harbitz,C.B.,Wynn,R.B.,Pedersen,G.,Løvholt,F.,2006.Submarinelandslides:process es,triggersandhazardprediction.Philos.Trans.:Math.Phys.Eng.Sci.364(1845),2009-2039.

McAdoo,B.G.,Pratson,L.F.,Orange,D.L.,2000.Submarinelandslidegeomorphology,US continentalslope. Mar.Geol.169,103-136.

Misra,A.A.,Sinha,N.,Mukherjee,S.,2015.Repeatridgejumpsandmicrocontinentseparation:insightsfr omNEArabianSea.Mar.Pet.Geol.59(1),406-428.

Mitchum,R.M.,Vail,P.R.,Sangree,J.B.,1977.Stratigraphicinterpretationofseismicreflectionpatternsin depositionalsequences.Part6.In:Payton,C.E.(Ed.),SeismicStratigraphyApplicationtoHydrocarbonExploration,8thed.AAPG,Tulsa,Okla,pp.117-133.

Morley,C.K.,2009.Growthoffoldsinadeepwatersetting.Geosphere5,59-89. 
Morley,C.K.,King, R., Hillis, R., Tingay,M.,Backe,G.,2011. Deepwaterfold

andthrustbeltclassification,tectonics,structureandhydrocarbonprospectivity:areview.EarthSci.Rev .104(1-3),41-91.

Moscardelli,L.,Wood,L.,2008.NewclassificationsystemformasstransportcomplexesinoffshoreTrinida d.BasinRes.20,73-98.

Moscardelli,L.,Wood,L.,2015.Morphometryofmasstransportdepositsasapredictivetool.Geol.Soc.Am .Bull.31221.1.

Naini,B.R.,Kolla,V.,1982.Acousticcharacterandthicknessofsedimentsof thelndusFan and thecontinentalmarginofwestern India. Mar. Geol.47(3-4),181-195.

Naini,B.R.,Talwani,M.,1982.Structuralframeworkandtheevolutionaryhistoryofthecontinentalmargino fwesternIndia.In:StudiesinContinentalMarginGeology,vol.34,pp.167-191.

Normark,W.R.,Moore,J.G.,Torresan,M.E.,1993.Giantvolcanorelatedlandslidesand the developmentofthe Hawaiianlslands.In:Schwab,W.C.,Lee, H.J., Twichell,D.C.(Eds.),SubmarineLandslides:SelectedStudiesintheU.S.ExclusiveEconomicZo ne.U.S.Geol.Surv.Bull.,184-196.

Okal, E.A., Synolakis,C.E., 2008.Farfieldtsunamihazardfrommegathrustearth quakesinthelndianOcean.Geophys.J.Int.172,995-1015.

Pandey,D.K.,Clift,P.D.,Kulhanek,D.K., andtheExpedition355Scientists,2015.Expedition355Prelimina ryReport:ArabianSeaMonsoon.InternationalOceanDiscoveryProgram.http://dx.doi.org10.2204/iod p.pr.355.2015.

Piper,D.J.W.,Shor,A.N.,HughesClarke,J.E., 1988.The 1929GrandBanks earth quake,slumpandturbiditycurrent.In:Clifton,H.E.(Ed.),SedimentologicalConsequencesofConvulsive GeologicEvents.Spec.Pap.,Geol.Soc.Am.229,77-92.Piper,D.J.W., Pirmez, C., Manley,P.L.,Long,D., Flood,

R.D.,Normark,W.R.,Showers,W.,1997.MasstransportdepositsoftheAmazonFan.In:Proceedingsofthe OceanDrillingProgram.ScientificResults,vol.155,pp.109-146.OceanDrilling Program.

Popenoe,P.,Schmuck,E.A.,Dillon,W.P.,1993.TheCapeFearLandslide:slopefailureassociatedwithsalt diapirismandgashydratedecomposition.In:Schwab,W.C.,Lee,H.J.,Twichell,D.C.(Eds.),Submarine Landslides:SelectedStudiesintheU.S.ExclusiveEconomicZone.U.S.Geol.Surv.Bull.,40-53.

Prior,D.B.,Bornhold,B.D.,Johns,M.W.,1984.Depositionalcharacteristicsofasub marinedebrisflow.J.Geol.92,707-727.

Rao,D.G.,1991.AmodelofbasementstructurefrommagneticanomaliesoftheBombayHighOilFiledwest ofIndia.Mar.Geol.96(1-2),111-122.

Rao,G.N.,2001.Sedimentation,stratigraphy,andpetroleumpotentialofKrishnaGodavariBasin,EastCoastofIndia.Am.Assoc.Pet.Geol.Bull.85,1623-1643.

Rao,A.,Hanumantha,Y., Subrahmanyam, C.,Rastogi,A.,Deka,B.,2002.Slopefailuresalong the westerncontinentalmarginof India: a consequenceofgashydratedissociation,rapidsedimentationrate, andseismicactivity?GeoMar.Lett.2 
2(3),162-169.

Steffens,G.S.,Biegert,E.K.,Sumner,H.S.,Bird,D.,2003.Quantitativebathymetricanalysesofselectedd eepwatersiliciclasticmargins:receivingbasinconfigurationsfordeepwaterfansystems.Mar.Pet.Geol .20(6-8),547-561.

Todal,A.,Edholm,O.,1998.ContinentalmarginoffwesternlndiaandDeccanlargeigneousprovince.Mar. Geophys.Res.20(4),273-291.

Torelli,L.,Sartori,R.,Zitellini,N.,1997.Thegiant chaoticbodyin the

AtlanticOceanoffGibraltar:newresultsfromadeepseismicreflectionsurvey.Mar.Pet.Geol.

$14(2), 125-134$.

Vanneste,M.,Mienert,J.,Bünz,S.,2006.TheHinlopenSlide:agiant,submarineslopefailureonthenorthe rnSvalbardmargin,ArcticOcean.EarthPlanet.Sci.Lett.245(1-2),373-388.

Wellman,P.,McElhinny,M.W.,1970.K-ArageoftheDeccantraps,India.Nature227,595-596.

Whiting,B.M.,Karner,G.D.,Driscoll,N.W.,1994.Flexuralstrengthandstratigraphicdevelopmentofthew estIndiancontinentalmargin.J.Geophys.Res.99,13791-13811.

Woodcock,N.H.,1979.Sizesofsubmarineslidesand theirsignificance.J.Struct.

Geol.1(2), 137-142.

Yamada,Y.,Kawamura,K., Ikehara,K.,Ogawa,Y.,Urgeles,R.,Mosher,D.,Chaytor,J.,Strasser,M.,2012. Submarinemassmovementsandtheirconsequences.In:Yamada,Y.,etal.(Eds.),SubmarineMassM ovementsandTheirConsequences.In:AdvancesinNaturalandTechnologicalHazardResearch,vol.3 1.Springer,Netherlands,pp.1-12. 